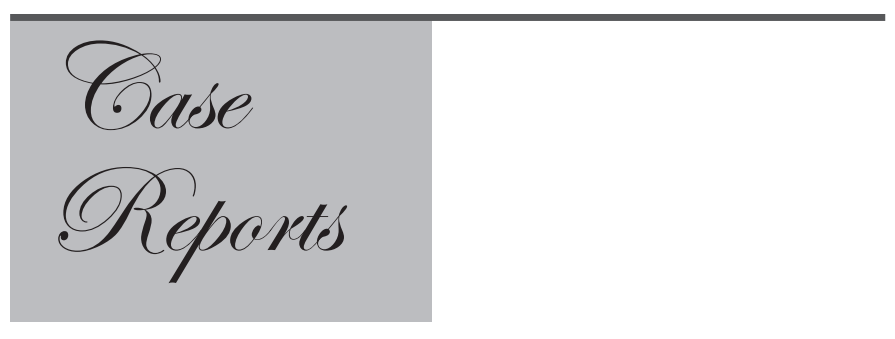

\section{Amoebic Liver Abscess: An Unusual cause of Isolated Dysphagia}

Amoebic Liver Abscess (ALA) is most common extraintestinal site of Entamoeba histolyticainfections, but is seen in $<1 \%$ of clinical infections. ${ }^{1}$ It is usually single and most commonly found in the right lobe of liver. The incidence of left lobe abscess (LLA) ranges from $5 \%$ to $21 \%$. LLA are treated with a lower threshold for aspiration because of higher risk of rupture. ${ }^{2}$ Its rupture superiorly causes pericarditis or pleuro-pulmonary involvement and rupture inferiorly causes peritoneal contamination of greater or lesser sac, and rarely leads to dysphagia as well. ${ }^{3}$ Management of a ruptured ALA with such complication has traditionally been surgery. We herewith present a case of ALA presenting with dysphagia one month after apparently successful medical treatment. The case was unique in the absence of pleural or pericardial involvement and successful management by percutaneous drainage (PCD).

\section{Case Report}

Forty-year male presented to an outside clinic with ten days history of fever which responded to antibiotics. He developed dysphagia for solids five days after defervescence, and he was able to swallow liquids with pain. He had dull upper abdominal pain and constipation. There were no respiratory symptoms, change of voice or cough on swallowing.
USG Abdomen showed a LLA with probable extension to the surrounding area. Subsequent endoscopy showed normal esophagus and a bulge in cardia of stomach (Figure 1). A diagnosis of Gastrointestinal Stromal Tumor (GIST) could not be ruled out.

Dysphagia progressed over the next two days from Grade 3 to 4 . At presentation to our hospital he had recurrent vomiting and severe pain on swallowing saliva. There was no pain abdomen or breathlessness. He appeared toxic and dehydrated. On examination he had fever, tachycardia without distended neck veins. Abdominal examination showed palpable tender left lobe of liver ( $5 \mathrm{~cm}$ in epigastrium). There was no guarding/ rigidity or shifting dullness, and normal bowel sounds were present. Chest examination was normal.

He gave a history of fever and abdominal pain about one month back, when he had a $41 \mathrm{ml}$ LLA on USG. He received antibiotic treatment for seven days after which he became afebrile and the treatment was stopped. He remained symptom-free till the present episode.

Investigation revealed mild anemia, neutrophilic leukocytosis, normal liver and kidney function. Chest X-ray was normal. IgG ELISA for E Histolytica (AccuDiagTM, Cortez Diagnostics) was positive (more than 0.5 OD). USG abdomen showedan abscess cavity of $85 \mathrm{ml}$ in the caudate lobe of liver (Figure 2). The cavity showed evidence of subcapsular leak posteriorly. A single pocket of fluid collection measuring $53 \mathrm{ml}$ was noted extending close to the GE junction.

$\mathrm{He}$ was treated with intravenous fluids, antibiotics, and analgesics. Under the USG guidance, two separate Malecotcatheters (10F, $24 \mathrm{~cm}$ ) with indigenously made side-holes were placed in the abscess cavity. Thick anchovy sauce like fluid was aspirated through the catheter. The dysphagia decreased to Grade 3. He was able to take food and water sufficiently by the next morning. The catheter was removed after 48 hours of complete resolution of collection and the cavity. He was discharged on Day 3 on oral metronidazole.

\section{Discussion}

ALA is a common infection in tropics, it responds well to treatment with metronidazole. It can extend along its 


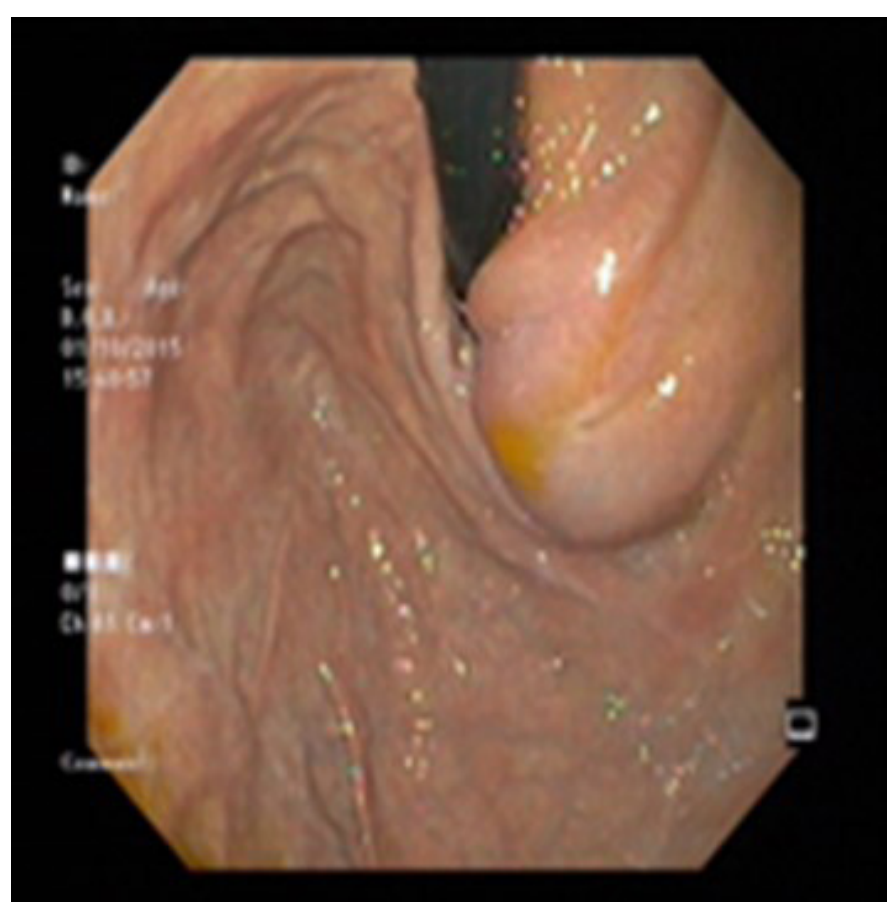

Figure 1: Endoscopic image of stomach cardia showing a boggy swelling.

periphery and can rupture into the thoracic structures like pleural or pericardial cavities and abdominal structures like stomach or peritoneal cavities, which has significant associated morbidity and mortality.

Our case presented one month after apparently successful treatment. The probable reasons could be improper anti-amoebic treatment, abscess refractory to treatment or secondary infection of the preexisting abscess cavity. ${ }^{5}$ The present case reemphasizes a need to have reimaging with USG and aspiration of the abscess if fever is not responding, recurs or the patient develops localizing signs. It also emphasizes the role of adherence to appropriate antiamoebictreatment.

Dysphagia is a well known but rare complication of rupture of left lobe abscess. In cases described it is usually associated with the involvement of pleural or mediastinal structures. Rudra et al. ${ }^{3}$ described two cases of ruptured Liver abscesses compressing gastric fundus, one of them had jaundice and communication with stomach, and the other one had left pleural effusion. Both were operated with a suspicion of a tumor, and one of them died post-surgery. Chandran et al. ${ }^{6}$ in their report described a case of progressive dysphagia where the patient had

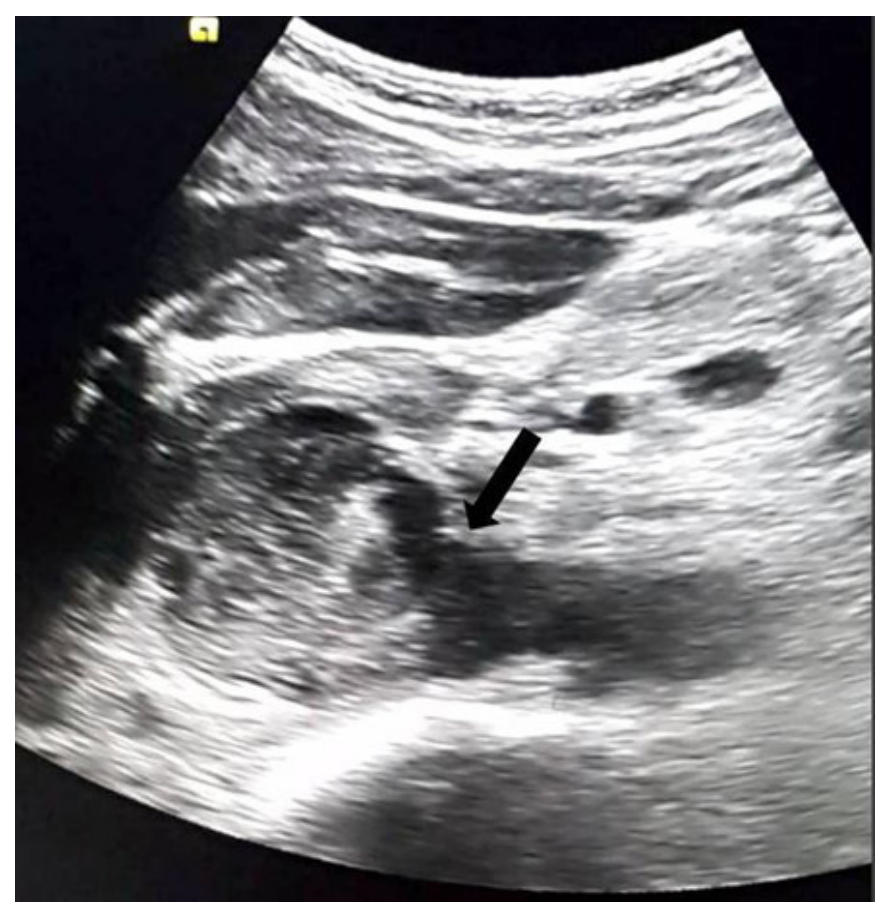

Figure 2: USG image showing left lobe abscess (arrow showing communication).

jaundice and pericardial tamponade, the patient needed emergency surgery and two weeks of hospital stay.

SANJEEV K THAKUR $^{1}$
RAJEEV PRIYADARSHI ${ }^{2}$
ANANT KUMAR
VIJAY PRAKASH

${ }^{1}$ Gastro Care \& Bihar Institute of Gastroenterology, Patna, Bihar, India

${ }^{2}$ Department of Radiodiagnosis, Indira Gandhi Institute of Medical Sciences, Patna, Bihar, India

${ }^{3}$ Arvind Hospital, Ashok Rajpath, Patna, Bihar, India

${ }^{4}$ Patna Medical College \& Hospital, Patna, Bihar, India

Corresponding Author: Dr Sanjeev K Thakur

Email: sanjeevtk02@yahoo.co.in

\section{References}

1. Peters RS, et al. Amoebic liver disease. Ann Rev Med 1982; 32:161-74.

2. Lamont N. M, Pooler N.B. Hepatic Amoebiasis. A study of 250 cases. Quart J Medicine 1958, 27,389. 
3. Rudra R, Shelton E. Left lobe amoebic liver abscess. Postgraduate Medical Journal 1976; 52, 269-274.

4. Coia, LR, Myerson, RJ, Tepper, JE. Late effects of radiation therapy on the gastrointestinal tract. Int J Radiat Oncol Biol Phys 1995; 31:1213

5. Thompson JE, Jr, Forlenza S, Verma R. Amebic liver abscess: a therapeutic approach. Rev Infect Dis. 1985; 7:171-179

6. Chandran SR, Balakrishnan RK, Chinnasamy B, Srinivasan GK. Amebic Hepatopericardial fistula causing cardiac tamponade. Ann Trop Med Public Health 2012; 5:387-9.

\section{Fibrovascular Polyp of the Esophagus: A Case Series}

Fibrovascular polyp of the esophagus is a rare condition causing dysphagia, and only a few cases have been reported in the literature. Clinical symptoms vary from difficulty in swallowing to fatal asphyxiation or an incidental finding on upper gastrointestinal endoscopy. Usually, polyps are intraluminal and polypoidal lesions which arise from the cervical part of the esophagus.

\section{Case Series}

Two of the patient presented with insidious onset of progressive dysphagia over two years while the other two had recurrent respiratory infection and vomiting respectively. On Upper gastrointestinal endoscopy (UGIE), there was a pedunculated polypoidal structure in the lumen, with attachment to the wall just below the upper esophageal sphincter. A barium swallow was done in two of the patients which showed smooth lesions (Figure 1). One of them used to vomit the mass frequently and swallow it, however, the other patient who regurgitated a polypoidal mass through the mouth, after UGIE which was partially excised and reduced under anesthesia (Figure 2). Computed tomography of the neck and thorax revealed a polypoidal mass in the lumen of lower cervical esophagus (Figure 3).

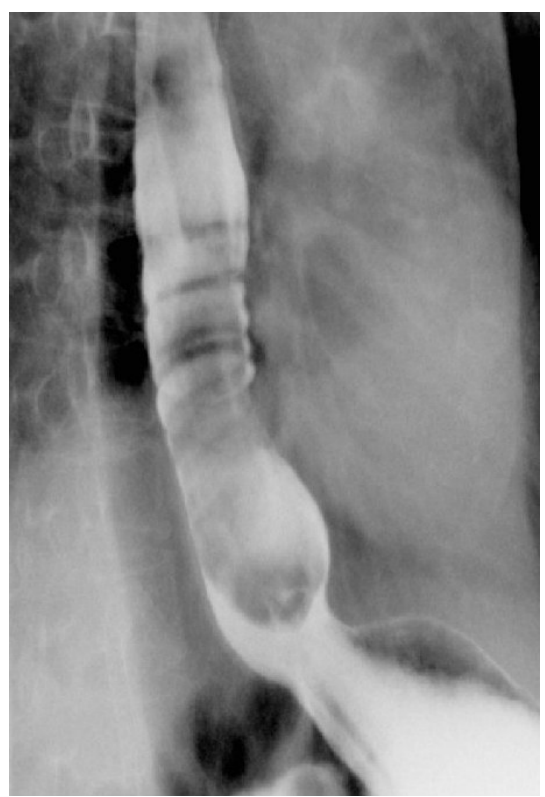

Figure 1: Dilated thoracic esophagus with smooth long filling defect which extends till the GE junction.

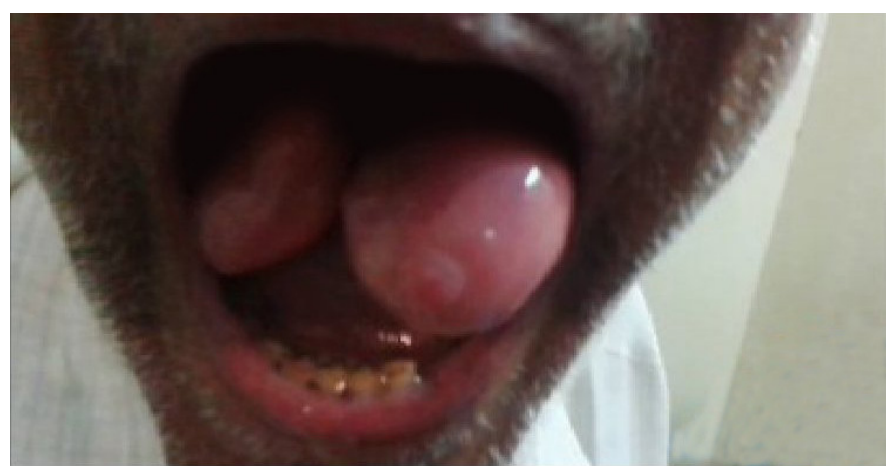

Figure 2: Regurgitation of the polyp.

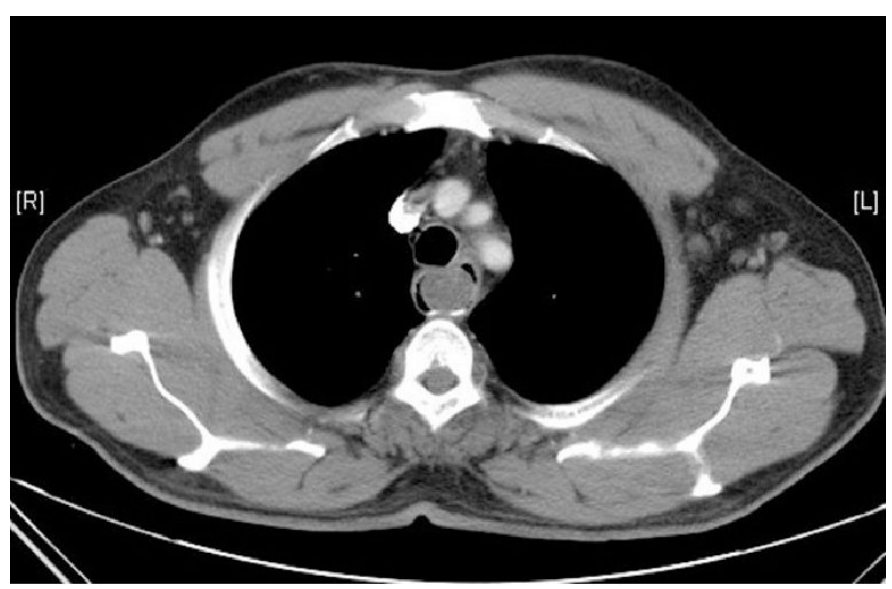

Figure 3: Large elongated polypoid soft tissue density lesion causing luminal narrowing. 